\title{
Conocimientos y hábitos alimentarios en mujeres diagnosticadas con hipertensión arterial del municipio de Sonsón - Colombia
}

\author{
N utrition knowledge and eating habits in women \\ diagnosed with hypertension in the municipality \\ of Sonsón - Colombia
}

\begin{abstract}
Arterial hypertension is a problem for the world public health as it carries, in most cases, cardiovascular, kidney and brain diseases. The objective of this research was to understand the knowledge and eating habits of a group of women diagnosed with hypertension in the municipality of Sonsón-Antioquia. Subjects and methods: The approach used was qualitative, which allowed us to understand the social and cultural factors that influence the feeding of this group of women. The method was ethnography; the techniques of data collection were individual and group interviews. Results: The food changes proposed to the diagnosed patients with arterial hypertension by the health personnel are oriented to reducing foods high in sodium and salt, but these recommendations are difficult to abide due to factors like food taste. The feeding recommendations offered by the health personnel are framed within nutritional issues that do not always consider the taste or the meanings attributed to food, nor the frequency of their consumption.
\end{abstract}

Key words: Municipality of Sonsón, eating habits, food consumption, arterial hypertension.

\section{INTRODUCCIÓN}

La hipertensión arterial (HTA) es considerada a nivel mundial como un problema de salud pública porque conlleva, en la mayoría de los casos, a enfermedades cardiovasculares, renales y cerebrales (1-3). Además, su prevalencia ha estado en aumento en los últimos años, posicionándose como una de las principales causas de muerte en todo el mundo (1), causando 9,4 millones de muertes anuales; para el 2008, la OM S (1) estimó una prevalencia global de hipertensión de $40 \%$ en adultos mayores de 25 años.

Colombia no es ajena a esta situación, en 2005 la prevalencia de HTA en personas entre 18 y 64 años de edad era $9,4 \%$ (4); la prevalencia actual es 9,97\%, mostrando un aumento de $0,57 \%$ en los períodos de 2005 al 2011 (5). En el departamento de Antioquia el año 2012 se reportaron 856.000 casos, presentándose una prevalencia en aumento en la población con mayor edad, en hombres y en las zonas urbanas (6). En el municipio de Sonsón-Antioquia, se observó que entre los años 2005 y 2011, las principales causas de mortalidad fueron por enfermedades del sistema circulatorio, presentándose un aumento en el año 2011 con 234.4 muertes por cada 100.000 habitantes; y afectando principalmente, a la población más envejecida (7).
Luz Marina Arboleda M. John Edinson Velásquez V.

Escuela de N utrición y D ietética, U niversidad de Antioquia Medellín, Colombia

Dirigir la correspondencia a: Profesora

Luz Marina Arboleda Montoya Carrera 75 \# 65-87 Bloque 44 Medellín-Colombia

Teléfono: (57) 2199222 Cel.: 3147728822

E-mail: luzmar.arboleda@gmail.com

Este trabajo fue recibido el 29 de Abril de 2015 y aceptado para ser publicado el 5 de Septiembre de 2015.

Entre los principales factores de riesgo para la aparición de enfermedades crónicas como la HTA, se encuentran la obesidad, el alcoholismo, el tabaquismo y el estrés, entre otros, que contribuyen al aumento de las cifras a nivel mundial y local; los cuales se presentan por una diversidad de dinámicas sociales tales como la disminución de tiempo dedicado a la familia, la disminución de la actividad física y el cambio de la alimentación tradicional por productos casi listos para el consumo, los cuales contienen gran cantidad de sodio y otros nutrientes nocivos para la salud como azúcares y grasas trans adicionados (8).

Estos cambios alimentarios producidos en las sociedades contribuyen a aumentar significativamente la presión arterial (9), lo cual se ha evidenciado en estudios como los reportados por Singer el 2015 (10), Taylor el 2011 (11) y Bravo (12) y Forman (13) el 2012.

En un estudio realizado en Colombia sobre los alimentos más vendidos en los supermercados y tiendas de barrio, se encontró que estos eran los que mayor contenido de sodio presentaban, como condimentos, salsas y aderezos, comidas listas para consumir y carnes frías, entre otros (14).

De acuerdo a lo anteriormente señalado, la alimentación es uno de los principales factores de riesgo que condi- 
ciona el aumento de la prevalencia de la HTA; sin embargo, el acto alimentario es un acto que por ser social y complejo es difícil de cambiar, pues a su alrededor se tejen sentidos y significados; por eso, es de suma importancia comprender las categorías simbólicas y sociocultural es de la alimentación para así establecer intervenciones pertinentes, humanas y de acuerdo a las realidades humanas.

De esta manera, tratando de comprender dichas categorías, en esta investigación se procuró interpretar los conocimientos y hábitos alimentarios en un grupo de mujeres diagnosticadas con hipertensión arterial en el municipio de Sonsón-Antioquia, el cual fue elegido por ser uno de los que presenta la mayor prevalencia de HTA en el departamento de Antioquia.

\section{SUJETOS Y MÉTODOS}

\section{Población y muestra}

La investigación se desarrolló en el municipio de Sonsón ubicado en el oriente Antioqueño, el cual tiene una población de 36.104 habitantes aproximadamente (15), de los cuales 15.221 pertenecen a la cabecera municipal y 20.883 a la zona rural.

El trabajo de campo se realizó con mujeres entre los 30 y 60 años de edad diagnosticadas con hipertensión arterial del área rural y urbana del municipio, pertenecientes a los diferentes niveles de SISBEN ${ }^{1}$. Entre los criterios de inclusión se consideraron que fueran habitantes del municipio y diagnosticadas con hipertensión arterial con un tiempo igual o superior a 5 años, para identificar los cambios en la alimentación.

\section{M etodología}

El enfoque utilizado fue cualitativo, que tiene como característica describir la realidad social a través de las percepciones y vivencias de la población que está siendo estudiada (16). El método que se utilizó fue la etnografía, definida como la descripción de los estilos de vida de un grupo de personas que comparten costumbres y entornos (17), como el grupo de mujeres de Sonsón participantes, que comparten el territorio local y las costumbres alimentarias.

Las técnicas que se emplearon para la recolección de la información fueron las entrevistas individuales y grupales, realizadas durante los meses de enero y febrero del año 2015. La planeación de las técnicas se hizo buscando la representatividad de mujeres de la zona rural y urbana tanto en las técnicas individuales como grupales. En total se realizaron dos entrevistas grupales conformadas cada una por cuatro mujeres, una de estas entrevistas se realizó en la zona rural y la otra en la urbana o cabecera del municipio; además se realizaron nueve entrevistas individuales, cuatro con mujeres de la zona urbana y cinco de la zona rural. Participando, de esta manera, diecisiete mujeres, nueve de la zona rural y ocho de la cabecera municipal de Sonsón.

Para llevar a cabo estas técnicas, se diseñó un guion de entrevista que giró en torno a la reflexión sobre el significado atribuido a la enfermedad y a la alimentación, al consumo de sal y caldos concentrados, y al papel que juega el personal de salud a la hora de brindar recomendaciones para mejorar el estado de salud.

\section{CONSIDERACIONES ÉTICAS}

La presente investigación no presentó riesgo para la población, según lo estipulado por la Resolución 008430 de 1993 del M inisterio de Salud de la República de Colombia.

Las mujeres participaron de manera voluntaria en las diferentes técnicas, firmaron un consentimiento informado, donde se les informó el objetivo de la investigación, se les explicó que en cualquier momento de la conversación podían interrumpir y pedir aclaraciones, y que no estaban obligadas a participar durante toda la conversación, además se les aclaró que durante el análisis de la información y escritura de los resultados sus nombres serían sustituidos por códigos para garantizar el anonimato. Y por último, se les pidió la aprobación para grabar las conversaciones generadas durante el desarrollo de las diferentes técnicas.

\section{PROCESAM IENTO DE LA INFORM ACIÓN}

Una vez finalizada la recolección de información, se procedió a transcribirla de forma literal, para iniciar el proceso de codificación de los datos en el cual se identificaron las unidades con sentido, que luego se integraron en cuerpos temáticos o categorías. Lo anterior se realizó utilizando una matriz de Excel versión 2010.

Luego se procedió a describir cada categoría, lo cual permitió tener una visión más amplia y comprensiva del fenómeno alimentario investigado. Posteriormente se trianguló la información, proceso que consistió en cotejar los resultados obtenidos con resultados de otras investigaciones, con teorías y con la visión de los investigadores. El proceso de análisis permitió identificar tres categorías, las cuales se describirán e interpretarán a continuación:

- $\quad$ Fuentes de información y conocimientos sobre la hipertensión arterial.

- Consumo alimentario y composición de las comidas.

- Cambios alimentarios y sus repercusiones familiares y sociales.

\section{RESULTADOS 2}

Fuentes de información y conocimientos sobre HTA

Las mujeres desconocían las causas y síntomas de la HTA y los motivos por los cuales la sal es inadecuada cuando se presenta esta enfermedad; de otro lado, conocían bastante las consecuencias relacionadas con las alteraciones cardíacas que produce la HTA.

Las participantes refieren que la información que obtienen sobre la enfermedad la reciben principalmente por el médico tratante y la enfermera que los atiende en los controles de la HTA en el Hospital San Juan de Dios del M unicipio de Sonsón. La orientación de estos profesionales se encamina a brindar recomendaciones tendientes a promover la actividad física, la alimentación saludable y la disminución de peso corporal. Respecto a la actividad física, les recomiendan que realicen caminatas diarias, o que integren este componente en las actividades diarias. Las recomendaciones alimentarias las orientan a controlar el consumo de alimentos ricos en grasas y sal/sodio, y a aumentar el consumo de frutas y verduras. Sin embargo, en estas recomendaciones no se brindaban las estrategias específicas para que ellas puedan llevar a cabo dichos cambios alimentarios, pues no se les brindaba las al-

1. Sistema de identificación de Potenciales Beneficiarios de Programas Sociales en Colombia.

2. En el Anexo A. Categorías y testimonios, se encuentran las conversaciones más relevantes de las participantes, en cada categoría del estudio. 
ternativas de sustitución de la sal, ni de la implementación de otros métodos de cocción diferentes a la fritura.

Otras fuentes de información que juegan un papel importante en la educación de las mujeres, son los medios masivos de comunicación como la radio y la televisión, donde presentan programas informativos y educativos que les brinda herramientas para el manejo de la enfermedad; además, utilizan otros medios como la internet y las revistas.

Consumo alimentario y composición de las comidas

La alimentación en la mayoría de los hogares de las participantes, conserva las características de una alimentación tradicional propia de la región, donde predomina un gusto marcado por cereales como la $\operatorname{arepa}^{3}$ y el arroz, sustitutos como el huevo y quesito, y bebidas tradicionales como el agua de panela ${ }^{4}$ y el chocolate.

Las mujeres tienen preferencias por alimentos ricos en carbohidratos como la papa, yuca, plátano, galletas, tostadas, panes y principalmente cereales como el arroz; también tienen un alto consumo de grasas, presentes en las preparaciones fritas; y un alto consumo de sodio, por el uso frecuente de caldos concentrados para sazonar los alimentos.

Las principales preparaciones consumidas en los seis momentos de comida en los hogares de las entrevistadas son:

El desayuno consumido con más frecuencia está compuesto por alimentos como arepa, arroz, quesito, huevo y galletas, tostadas o panes, alimentos a los que llaman "parva", que acompañan con bebidas tradicionales como el chocolate, agua de panela o café con leche.

En la media mañana, entrecomida poco usual en el municipio, prefieren alimentos como frutas, galletas, jugo de fruta o tinto ${ }^{5}$.

El almuerzo usualmente es un plato de $\operatorname{seco}^{6} \mathrm{y}$, en ocasiones, otro de sopa. El seco está compuesto por preparaciones como arroz, carne de res, cerdo, pollo, o pescado, tajadas de papa o plátano. El plato de sopa puede ser de pasta, papa, frijoles o lentejas, y al finalizar, toman un juego de fruta, "en el almuerzo me tomo un jugo puede ser con una tajadita de plátano, unas cucharaditas de arroz y una carnita, muy de vez en cuando como sopas, lentejas y frijoles"7. En otras ocasiones, consumen a la hora del almuerzo platos tan tradicionales como sancocho ${ }^{8}$ o sudado ${ }^{9}$.

A diferencia de la media mañana, el algo es más consumido en los hogares de las participantes, ya que, según ellas, este "quiebra" el ayuno entre el almuerzo y la comida. Entre los alimentos más consumidos a esta hora del día están las bebidas tradicionales, como tinto, chocolate, café con leche, las cuales se acompañan con un cereal como galletas, tostadas o arepa y un sustituto que generalmente es queso, quesito o huevo.

La composición de la comida es muy similar a la del desayuno, constituida por alimentos como arepa y otros cereales como galletas, tostadas o panes, y sustitutos como queso, quesito o huevo, que acompañan con chocolate, café con leche $o$ agua de panela.

\section{Cambios alimentarios y sus repercusiones familiares y sociales}

Muchas de las mujeres han cambiado sus hábitos alimentarios, acatando las recomendaciones dadas por el personal de salud, a pesar de estar alejados de sus gustos alimentarios. Los principales cambios se relacionan con la disminución en el consumo de alimentos fritos, azúcares y sal agregada, y con el aumento del consumo de frutas. Las familias también han cambiado algunos hábitos alimentarios de manera voluntaria y por solidaridad, principalmente lo que respecta la disminución del consumo de sal.

No obstante, hay cambios que las participantes no han podido llevar a cabo, como es la disminución del consumo de caldos concentrados, por considerar que los alimentos no quedan con el mismo sabor. Fueron enfáticas en afirmar que el uso de la sal es de suma importancia para mejorar el sabor de las comidas, y que su ausencia hace que la comida sea insípida y brinde poca sensación de saciedad. Aunque dicen que es muy difícil acostumbrarse a las comidas simples, las consumen así por su salud.

Es de aclarar, que dichos cambios se establecen en el ámbito privado e íntimo del hogar, donde ellas tienen libertad de elección y de preparación, pero ya en el ámbito social y comunitario, se les hace más difícil llevar a cabo estos cambios alimentarios por la cultura propia del municipio, y por las reglas implícitas presentes en este acto, por ejemplo, no recibir los alimentos brindados, es mal visto por las personas de este territorio.

\section{DISCUSIÓN}

Los hábitos alimentarios inadecuados constituyen un factor de riesgo para el aumento de la prevalencia de la hipertensión arterial (1), no obstante, la complejidad implícita en el acto alimentario y las dimensiones simbólicas y culturales no siempre son reconocidas por el personal de salud, quienes realizan recomendaciones alimentarias y sugerencias difíciles de llevar a cabo porque comprometen uno de los aspecto más preciados de la alimentación, el sabor de los alimentos y preparaciones.

M encionado "sabor" está fuertemente relacionado con los gustos alimentarios, porque se forja desde la infancia. En una revisión sobre el tema para conocer los factores que determinan el gusto por lo salado, se encontró que la cultura, las costumbres familiares y los componentes genéticos son los principales factores que influyen en el gusto; además, se identificaron otros aspectos que intervienen en el gusto como el tipo de alimento, la temperatura, la textura y la presencia de otros ingredientes (18).

El sabor juega un papel preponderante a la hora de elegir alimentos; según un estudio sobre grasas en Costa Rica, se identificó que este nutriente se une con los condimentos y estimulan el olfato (19), lo cual hace más apetecibles las preparaciones; además, los alimentos grasos dan ciertas características organolépticas de textura, sabor, jugosidad y mordida crocante a los alimentos $(19,20)$, haciéndolos más apetecibles.

Aspectos como los anteriores, fueron los principales motivos de preferencia y elección en las mujeres participan-

\footnotetext{
3. Amasijo preparado con maíz.

4. Bebida a base de caña de azúcar, utilizada en la zonas rurales y urbanas de Colombia para acompañar las comidas.

5. Infusión de café en agua, utilizada en las comidas intermedias en Colombia.

6. Seco: nominación que se le da en Antioquia a un plato conformado por preparaciones sólidos.

7. Entrevista, mujer 3 enero de 2015.

8. Sancocho: nominación que se le da en Antioquia a un plato conformado por una sopa con mezcla de tubérculos.

9 Sudado: nominación que se le da en Antioquia al bistec.
} 
tes de Sonsón, quienes se inclinaban por alimentos salados y altos en grasas por dos motivos, primero, por corresponder con los sabores de las preparaciones tradicionales que acostumbran consumir y segundo, porque los alimentos como la sal y las grasas mejoran el sabor y las características organolépticas de las preparaciones y platos. Varias investigaciones, señalan que la compra de los caldos concentrados en los mercados colombianos está en aumento $(14,21)$, a pesar de ser uno de los causantes del aumento en las cifras de presión arterial en la población.

Se puede identificar que la alimentación es un acto cultural, revestido de simbolismos y recuerdos compartidos, aspectos que no pueden desconocerse al brindar educación nutricional por parte del personal de salud, pues dicho desconocimiento hace que esta educación sea descontextualizada. Investigaciones como la de Arboleda, concluyen que la gran influencia del modelo biomédico en la formación de profesionales del área de la salud, hace que estos consideren el saber popular molesto para su quehacer (22); así mismo, en otras investigaciones realizadas simultáneamente en Argentina, Costa Rica y Ecuador, concluyeron que el equipo de salud brinda poca información a los pacientes sobre temas relacionados al proceso salud-enfermedad, limitándose más a realizar labores técnicas y operativas, que a la educación de los paciente (23).

Un estudio de Oliveira y Noriega concluyó que la interacción entre el personal de salud y el paciente todavía está "centralizada en técnicas y tareas, que privilegian acciones de prescripción y de rutina, focalizadas en el cuerpo, en la enfermedad, en el tratamiento y en la adhesión" y no en el reconocimiento de los aspectos sociales y culturales (24).

\section{CONCLUSIONES}

- La alimentación en el municipio de Sonsón se caracteriza por ser tradicional de la cultura antioqueña, en la cual predomina la preferencia por alimentos como la arepa, el arroz y las tajadas de papa y plátano, y el alto uso de caldos concentrados y sal para preparar los alimentos.

- Los cambios alimentarios propuestos por el personal de salud están enmarcados en aspectos nutricionales, los cuales se orientan a la disminución de alimentos ricos en sodio, grasas y azúcares, sin considerar que son los alimentos de preferencia de la población del municipio. Las mujeres participantes de esta investigación tratan de disminuir el consumo de alimentos altos en sal y sodio, pero se les dificulta, porque es un sabor que hace parte de su referente cultural, el cual le da sentido y significado a su alimentación.

\section{RECOM ENDACIONES}

Cuando se fomenten cambios alimentarios, se debe partir del reconocimiento de los gustos y cultura alimentaria de los sujetos, proponer cambios graduales y brindarles herramientas prácticas para que logren implementar los cambios sin que se afecte el sabor de los alimentos y el gusto alimentario, y así, los sujetos van atribuyendo significados a las nuevas preparaciones y a los nuevos sabores, que ya empiezan a ser parte de su alimentación.

\section{RESUM EN}

La hipertensión arterial es un problema de salud pública mundial porque conlleva, en la mayoría de los casos, a enfermedades cardiovasculares, renales y cerebrales. El objetivo de esta investigación fue indagar sobre los conocimientos y hábitos alimentarios de un grupo de mujeres diagnosticadas con hipertensión arterial en el municipio de Sonsón-Antioquia. Sujetos y métodos: el enfoque utilizado fue cualitativo, el que permitió comprender los factores sociales y culturales que influyen en la alimentación de este grupo de mujeres. El método fue la etnografía; las técnicas de recolección de información fueron entrevistas individuales y grupales. Resultados: Los alimentos que más gustan en los hogares son los fritos, salados y condimentos procesados. Los cambios alimentarios propuestos por parte del personal de salud a las pacientes diagnosticadas con hipertensión arterial, se orientan a disminuir alimentos ricos en sodio y sal, lo cual les resulta difícil de llevar a cabo por factores culturales. Las recomendaciones alimentarias brindadas por el personal de salud se enmarcan en aspectos nutricionales que no siempre consideran los gustos alimentarios, ni los significados atribuidos a los alimentos.

Palabras clave: M unicipio de Sonsón, hábitos alimentarios, consumo alimentario, hipertensión arterial.

\section{BIBLIOGRAFIA}

1. Organización M undial de la Salud (OM S). Información general sobre la Hipertensión en el mundo, una enfermedad que mata en silencio, una crisis de salud pública mundial. Ginebra, Suiza; 2013.

2. González A. Hipertensión: alcance del Problema. [Internet].[Consultado el 2015 febrero 17]Disponible en:http:// www.sociedadmexicanadehipertension.mx/pdf/hipertensionalcance.pdf

3. Paul et al. 2014 Evidence-Based Guideline for the M anagement of High Blood Pressure in Adults Report From the Panel M embers Appointed to the Eighth Joint National Committee (J NC 8). ClinicalReview\&Education. 2013.

4. ICBF, Profamilia, Instituto Nacional de Salud, Universidad de Antioquia, OPS. Encuesta Nacional de la situación nutricional en Colombia (ENSIN). Bogotá; 2005.

5. República de Colombia. M inisterio de Salud. Análisis de Situación de Salud de Colombia.2013. Bogotá, Colombia; 2014.

6. Gobernación de Antioquia, gerencia de Comunicaciones. 7 de Abril, día mundial de la salud. [Internet].[Consultado el 2015 febrero 17]Disponible en:http://antioquia.gov. co/gobant/index.php/component/content/article/8sergio-fajardo/19-7-de-abril-dia-mundial-de-la-salud.

7. Situación de Salud con el M odelo de los Determinantes Sociales de Salud. M unicipio de Sonsón. Dirección Local de Salud de Sonsón, Sonsòn; 2013.

8. Cruz C. Consumo Alimentario: Causas y consecuencias para la salud. El Boletín. [Internet] [Consultado el 20 de Enero de 2014]. Disponible en: http://www.rebelion.org/ docs/145589.pd

9. Báez L. Guías Colombianas para el diagnóstico y tratamiento de la Hipertensión arterial. Rev. Colomb. Cardiol.2007;3(1):187-213.

10. Singer $P$, Cohen $H$, Alderman $M$. Assessing the Associations of Sodium Intake with Long-Term All-Cause and Cardiovascular M ortality in a Hypertensive Cohort. Am J Hyperten. 2015;28(3):335-42.

11. Taylor R, Ashton K, M oxham T, Hooper L, Ebrahim S. Reduced Dietary Salt for the Prevention of Cardiovascular Disease: A M eta-Analysis of Randomized Controlled Trials (Cochrane Review).Am J Hyperten. 2011; 24(8):843-53.

12. Bravo I, M ichea L. Análisis de la evidencia experimental que muestra efectos nocivos de la sal y su relación con 
la hipertensión arterial. M edwave 2012;12(2):1-5.

13. Forman J, Scheven L, Jong P, Bakker S, Curhan G, Gansevoort R. Association Between Sodium Intake and Change in Uric Acid, Urine Albumin Excretion, and the Risk of Developing Hypertension. Circulation 2012; 125: 3108-16.

14. Carmona IC, Gómez B, Gaitán D. Contenido de sodio en alimentos procesados comercializados en Colombia, según el etiquetado nutricional. Perspect Nutr Humana. 2013; 16:61-82.

15. M unicipio de Sonsón. [Internet]. [acceso 22 de Noviembre de 2014]. Disponible en: http://www.galeon.com/ antioquiaparaelmundo/sonson.html

16. Bonilla E, Rodríguez P. M anejo de los datos cualitativos. En: M ás allá del dilema de los métodos: la investigación en ciencias sociales. 3 ed. Bogotá: Norma; 1997; p. 243-310.

17. Franco Giraldo FA, Arboleda M ontoya LM. Aspectos socioculturales y técnico-nutricionales en la alimentación de un grupo de adultos mayores del centro gerontológico Colonia de Belencito de Medellín-Colombia. Perspect Nutr Humana. 2010; 12:61-74.

18. López NC. El gusto por el sabor salado. Perspect Nutr Humana. 2014;16: 99-109.

19. Aráuz A, Roselló M, Guzmán S, Padilla G. Validación de un cuestionario de hábitos alimentarios asociados al consumo de grasas y azúcares. ALAN . 2008; 58(4): 392-6.

20. Suaterna Hurtado AC. La fritura de los alimentos: el aceite de fritura. Perspect Nutr Humana. 2009; 11:39-53.

21. Botero M M , Abello Ramírez L, Chamorro López M, Torres Guette V. Factores compensatorios y no compensatorios que influyen en la decisión de compra de productos culinarios en la categoría de caldos concentrados, en consumidores de la ciudad de Barranquilla. Univ. Psychol.2005;4(3):393-402.

22. Arboleda LM. Significado del saber popular en alimentación en un grupo de nutricionistas dietistas. PerspectNutr Humana. 2007; 9:49-60.

23. Sánchez $G$, Peña L, Varea $S$, M ogrovejo $P$, Goetschel $M L$, M ontero-Campos M A, et al. Conocimientos, percepciones y comportamientos relacionados con el consumo de sal, la salud y el etiquetado nutricional en Argentina, Costa Rica y Ecuador. Rev Panam Salud Pública. 2012;32(4):259-64.

24. Reiners $A O$, Nogueira $M$. Concientización del usuario hipertenso sobre la adhesión al tratamiento. Rev. LatinoAm Enfermagem. 2009;17 (1):59-65.

Anexo A. Tabla 1. Categorías y testimonios

Fuentes de información y conocimientos sobre la hipertensión arterial
Consumo alimentario y composición de las comidas
Cambios alimentarios y sus repercusiones familiares y sociales
- "Pues a mí me duele la cabeza, me sube un calor horrible me dicen pues, que le puede dar a uno un derrame".

(Entrevista, mujer 6 enero de 2015).

- "Ah pues yo sé que es un riesgo, que puede darme un infarto cardiaco o un derrame".

(Entrevista, mujer 6 enero de 2015)

- "Si, yo estoy en control en el hospital, la enfermera es la que nos da la información y el médico".

(Entrevista, Grupo 2 enero de 2015).

- "Ah pues cuando yo entré donde el doctor ya él me explicó y me tomó la presión y me dice que mucho cuidado, que camine mucho..."

(Entrevista, Grupo 1 enero de 2015).

- "La enfermera me ha informado sobre los hábitos alimenticios, que me debo controlar mucho la sal, el azúcar y los alimentos con altas grasas, y bajar de peso".

(Entrevista, mujer 4 enero de 2015).

- "Pues que uno debe comer más simplecito y cuidarse mucho de la sal y las grasas". (Entrevista, mujer 1 enero de 2015).

- "Ah pues el médico, y uno a veces escucha cosas por la emisora, y uno escucha mucho". (Entrevista, mujer 5 enero de 2015)
- "Bueno yo normalmente, me tomo un cafecito con leche, arepita y una tajada de quesito y huevo".

(Entrevista, mujer 1 enero de 2015).

- "Para mí el desayuno es la arepita con huevo y quesito y tomo chocolate". (Entrevista, mujer 7 enero de 2015).

- "En la media mañana me puedo comer unas galletas o una frutica"10 "por allá las 10 u 11 me tomo una media mañana que puede ser una frutica". (Entrevista, mujer 2enero de 2015)

- "Al almuerzo, soy muy variada en eso, porque a veces si me hago mi ensalada, mi carne asada y porción de sopa; y hay veces... me como el sancochito". "En el algo me puedo comer una parvita con café". (Entrevista, Grupo 1 enero de 2015).

- "De algo como, chocolate, con pan, quesito, galletas, o arepa con huevo". (Entrevista, mujer 4 enero de 2015).

- "normalmente como arroz, con huevo revuelto, arepa chocolate".

(Entrevista, mujer 2 enero de 2015).

- "Pues normalmente me como una arepa con una tajada de queso y huevo y café con leche".

(Entrevista, mujer 6 enero de 2015).

- "En la comida consumo aguapanela, arroz, huevo y galletas o arepa".

(Entrevista, Grupo 2 enero de 2015).

- "Al almuerzo me como algunas veces unos frijoles, arroz, chicharrón, patacones ${ }^{13}$ fritos, papas fritas, ensalada. (...) otras veces me frito mi carnita".

(Entrevista, Grupo 1 enero de 2015).
- "Pues yo estoy comiendo más simple, aunque me ha dado duro, además, no como tantas harinas como antes, me estoy cuidando del frito que antes me gustaba mucho".

(Entrevista, mujer 1 enero de 2015).

- "Yo si he hecho algunos cambios, no salo doblemente los alimentos, con las carnes trato de que sean asadas siempre, no consumo chorizos, ni comidas chatarra.". (Entrevista, mujer 4 enero de 2015).

- "El cambio es de todos, porque cuando hay que hacer almuerzo, yo hago para todos".

(Entrevista, Grupo 1 enero de 2015).

- "Bueno, el consumo de sal es excesivo, porque yo siento que las comidas sin sal o sin los condimentos no saben lo mismo." (Entrevista, mujer 4 enero de 2015).

- "Me fascina el Maggi ${ }^{11}$, vea yo en el mercado echo quince pastas, si la sopa es poquita, le echo poquitica pasta".

- (Entrevista, mujer 8 enero de 2015).

- "...eso no falta, porque mi mamá dice que eso le da sazón al cocinar, entonces no falta el M aggi en la casa". (Entrevista, mujer 9 enero de 2015).

- "Horrible no puedo dejar de comer sin sal, la comida no sabe igual". (Entrevista, Grupo 1 enero de 2015).

- "Siempre es difícil y la comida no sabe igual".

(Entrevista, mujer 1 enero de 2015).

- "Uno se va acostumbrando pero al principio es difícil porque el sabor de la comida cambia mucho".

(Entrevista, mujer 4 enero de 2015).

Fuente:

10 Entrevista, Grupo 1 enero de 2015

11 Preparación a base de plátano, sometido a fritura.

12 Concentrado de gallina en polvo.

Elaboración propia a partir de la información obtenida. 